\title{
Predicting the risk of developing type 2 diabetes in Chinese people who have coronary heart disease and impaired glucose tolerance
}

\author{
Shishi Xu ${ }^{1,2}$ | | Charles A.B. Scott ${ }^{2} \quad$ | Ruth L. Coleman ${ }^{2}$ | \\ Jaakko Tuomilehto $^{3,4}$ | Rury R. Holman ${ }^{2}$
}

${ }^{1}$ Division of Endocrinology and Metabolism, West China Hospital, Sichuan University, Chengdu, China

${ }^{2}$ Diabetes Trials Unit, University of

Oxford, Oxford, UK

${ }^{3}$ Department of Public Health Solutions, National Institute for Health and Welfare, Helsinki, Finland

${ }^{4}$ Department of Public Health, University of Helsinki, Helsinki, Finland

\section{Correspondence}

Shishi Xu, Division of Endocrinology and Metabolism, West China Hospital,

Sichuan University, Chengdu, 610041,

China.

Email: shishixu@stu.scu.edu.cn

\begin{abstract}
Aims: Robust diabetes risk estimates in Asian patients with impaired glucose tolerance (IGT) and coronary heart disease (CHD) are lacking. We developed a Chinese type 2 diabetes risk calculator using Acarbose Cardiovascular Evaluation (ACE) trial data.

Methods: There were 3105 placebo-treated ACE participants with requisite data for model development. Clinically relevant variables, and those showing nominal univariate association with new-onset diabetes $(P<.10)$, were entered into BASIC (clinical variables only), EXTENDED (clinical variables plus routinely available laboratory results), and FULL (all candidate variables) logistic regression models. External validation was performed using the Luzhou prospective cohort of 1088 Chinese patients with IGT.

Results: Over median 5.0 years, 493 (15.9\%) ACE participants developed diabetes. Lower age, higher body mass index, and use of corticosteroids or thiazide diuretics were associated with higher diabetes risk. C-statistics for the BASIC (using these variables), EXTENDED (adding male sex, fasting plasma glucose, 2-hour glucose, and HbA1c), and FULL models were 0.610, 0.757, and 0.761 respectively. The EXTENDED model predicted a lower $13.9 \%$ 5-year diabetes risk in the Luzhou cohort than observed $(35.2 \%, 95 \%$ confidence interval $31.3 \%-39.5 \%$, C-statistic 0.643).
\end{abstract}

Conclusion: A risk prediction model using routinely available clinical variables can be used to estimate diabetes risk in Chinese people with CHD and IGT.

\section{KEYW O R D S}

coronary heart disease, impaired glucose tolerance, risk prediction, type 2 diabetes mellitus

\section{Highlights}

- We developed a 5-year diabetes risk calculator for Chinese people with CHD and IGT. 
- Higher FPG, 2hPG, and HbAlc were major determinants of new-onset diabetes.

- It can inform decision-making when considering primary prevention measures.

\section{1 | INTRODUCTION}

Primary prevention of type 2 diabetes (T2D) has become a major challenge, particularly for China, which has the world's largest number of people with diabetes (116.4 million cases in 2019) equating to one in four of the world's adults with diabetes. ${ }^{1}$ A major concern is that almost half of all adults in China - estimated to be 400 to 500 million people - have "prediabetes".,3 Identifying those individuals at highest risk of progressing to T2D to inform the need for early intervention is highly desirable, given that many trials have now shown that T2D can be delayed or prevented by lifestyle modification ${ }^{4-6}$ or by pharmacological agents, ${ }^{7-10}$ with dietary intervention in the Da Qing study also reducing cardiovascular and allcause mortality in the longer term. ${ }^{11}$

To date, few diabetes risk prediction tools have been tailored specifically for people with impaired glucose tolerance (IGT). The two that were derived from large-scale IGT trials ${ }^{12,13}$ used predominantly Caucasian cohorts and have not been evaluated in Chinese populations, which may well have different characteristics. ${ }^{14,15}$ Patients with established coronary heart disease (CHD) are of particular concern as they are at greater risk of developing T2D than the general population. Indeed, over half of all patients with CHD have been shown to have T2D or "prediabetes" in Western ${ }^{16}$ and Chinese ${ }^{17}$ cohorts attending cardiology outpatient clinics or admitted to hospital cardiovascular wards.

We have used data from the Acarbose Cardiovascular Evaluation (ACE) trial, which randomized Chinese patients with CHD and IGT to acarbose or placebo with prospective ascertainment of new-onset diabetes, ${ }^{10}$ to develop a T2D risk calculator for a Chinese population.

\section{2 | METHODS}

\section{1 | Population}

The ACE trial design, baseline population characteristics, and results have been published previously. ${ }^{10,18,19}$ Briefly, ACE was a randomized, double-blind, placebo-controlled, event-driven, Phase IV superiority trial conducted in 176 outpatient clinics in tier 3 and tier 2 hospitals in
China. Eligible participants were aged 50 years or older with established CHD (defined as previous myocardial infarction, previous unstable angina, or current stable angina), and IGT (confirmed by a $75 \mathrm{~g}$ oral glucose tolerance test [OGTT]). Between March 2009 and October 2015, 6522 patients were enrolled and included in the intention-to-treat population, with 3272 assigned at random to acarbose and 3250 to placebo. As acarbose has been shown to reduce the risk of $\mathrm{T} 2 \mathrm{D},{ }^{7,10}$ only the placebo group was considered in this analysis.

The protocol of ACE trial was approved by the University of Oxford Tropical Research Ethics Committee, and by central or local ethics committees (as appropriate) at participating sites. All participants provided written informed consent. The ACE trial was registered with ClinicalTrials.gov, number NCT00829660, and the International Standard Randomized Controlled Trial Number registry, number ISRCTN91899513.

\section{2 | Diagnosis of diabetes}

During the ACE trial, fasting plasma glucose (FPG) values were measured every 4 months and a $75 \mathrm{~g}$ OGTT was performed annually. If either of these tests suggested diabetes, a confirmatory OGTT was done. Progression to diabetes was considered to have occurred if an elevated FPG $(\geq 7.0 \mathrm{mmol} / \mathrm{L})$ and/or 2-hour plasma glucose ( $2 \mathrm{hPG})(\geq 11.1 \mathrm{mmol} / \mathrm{L})$ value were recorded on two consecutive study visits, or if a diagnosis of diabetes was made by a nontrial physician and confirmed subsequently by an independent adjudication committee masked to study therapy allocation. ${ }^{10}$

\section{3 | Risk factors for T2D}

T2D risk factors considered included baseline demographic, clinical, and laboratory variables, which were selected for their availability in routine clinical practice for patients with CHD or were variables used commonly in previous diabetes risk scores. All values used were taken from the screening visit, (except for HbA1c which was captured only at the randomization visit), as the ACE trial encouraged optimization of CHD risk 
reduction therapies during a 4-week run in the period before randomization. ${ }^{18}$

\section{$2.4 \quad$ Statistical analysis}

ACE participants were categorized according to whether or not they had progressed to diabetes by their end of follow-up. Baseline characteristics for progressors and nonprogressors were summarized by median and interquartile range for continuous variables and numbers and percentages for categorical variables. Continuous variables were compared using Wilcoxon rank sum tests, and categorical variables were compared using Pearson chisquare tests. All statistical analyses were performed using SAS (version 9.4$)^{20}$ or R (version 3.4.3). ${ }^{21}$

\section{5 | Development of 5-year T2D risk prediction models}

Three risk prediction models using ACE baseline data were developed using methodology aligned to the Transparent Reporting of a Multivariable Prediction Model for Individual Prognosis or Diagnosis (TRIPOD) statement ${ }^{22}$ : (i) a BASIC model, limited to demographic and readily available clinical characteristics; (ii) an EXTENDED model, which added routinely available laboratory variables to the BASIC model; and (iii) a FULL model, using all prespecified ACE baseline variables.

The precise dates of diabetes onset are not available as ACE participants were assessed only every 4 months for possible development of diabetes. Accordingly, we used logistic regression rather than Cox models to build our risk calculator. Multivariable models were built with candidate variables included only if considered to be clinically relevan, or showing a nominally significant $(P<0.10)$ univariate relationship with new-onset diabetes. Both forward and backward selection methods were used to eliminate nonsignificant variables. The principal criteria for selecting models were an improvement in the C-statistic (increment $\geq 0.005$ ) and goodness of fit $(P>0.05$ indicating good fit). Interactions between sex and other covariates were also tested.

\section{6 | Development of a 5-year T2D risk score}

For ease of use in everyday clinical practice a risk scoring algorithm was developed, based on the optimal prediction model with routinely available variables. Continuous variables were categorized by tertiles, quartiles or clinically relevant cut-points. For example, body mass index (BMI) was categorized by the overweight $(24 \mathrm{~kg} /$ $\left.\mathrm{m}^{2}\right)$ and obesity $\left(28 \mathrm{~kg} / \mathrm{m}^{2}\right)$ threshold values recommended by the Chinese guidelines. ${ }^{23}$ All variables were then entered into a logistic regression analysis to compute $\beta$ coefficients so that each could be assigned an estimated risk score. The sum of these scores was used to derive an estimated 5-year risk of new-onset diabetes, which in turn could be classified as "Modest" (0\%-25\%), "Moderate" (25\%-50\%), or "High" (>50\%), as described previously. $^{12}$

\subsection{Internal validation}

The performance of the three 5-year diabetes risk prediction models and the 5-year diabetes risk score in terms of discrimination (whether they distinguished between people who did/did not develop diabetes) and calibration (extent to which predicted probabilities agreed with the observed risk across groups of individuals) was evaluated in ACE placebo participants. The Cstatistic was used to assess discrimination, which was classified as poor $(0.5$ to $<0.6)$, acceptable ( 0.6 to 0.7 ), good $(0.7$ to $<0.8)$, very good $(0.8$ to $<0.9)$, or excellent $(\geq 0.9)$. Calibration was estimated using the HosmerLemeshow test, with a good fit indicated by a $P$ value $>0.05$, and graphically by comparing the predicted probability against the observed probability across deciles of predicted risk.

\section{8 $\quad$ External validation}

External validation of the diabetes prediction models and the risk score algorithm were performed using data from the Luzhou survey, an independent prospective Chinese cohort followed between 2011 and 2016. This survey was part of the Risk Evaluation of cAncers in Chinese diabeTic Individuals: a lONgitudinal (REACTION) study, a multicenter prospective observational study investigating the association between diabetes and the risk of cancer among individuals with or without T2D in mainland China. ${ }^{24} \mathrm{~A}$ total of 10007 residents aged between 40 and 89 years were enrolled from five Luzhou communities using a multistage cluster random sampling method, of which 2565 had IGT (with or without CHD). In the Luzhou survey cohort, incident diabetes was defined as any one, or a combination, of FPG $\geq 7.0 \mathrm{mmol} / \mathrm{L}, 2 \mathrm{hPG} \geq 11.1 \mathrm{mmol} / \mathrm{L}$, or a self-report of a previous diagnosis of T2D made by a health care professional. In 2014 and 2016, 1155 of these IGT participants were reviewed to determine if they had developed 
T A B LE 1 Acarbose Cardiovascular Evaluation (ACE) participants baseline characteristics by progression or not to diabetes

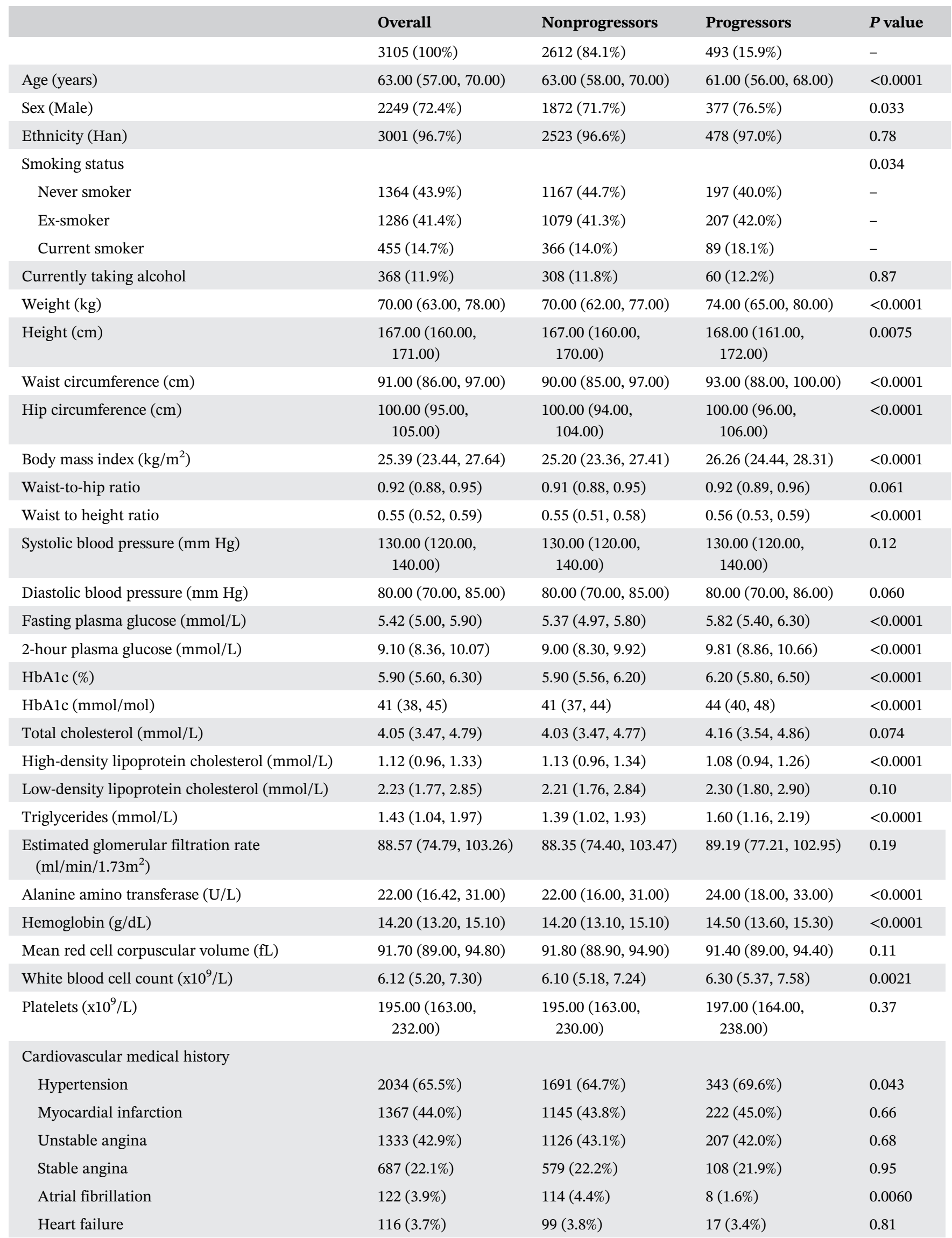


TABLE 1 (Continued)

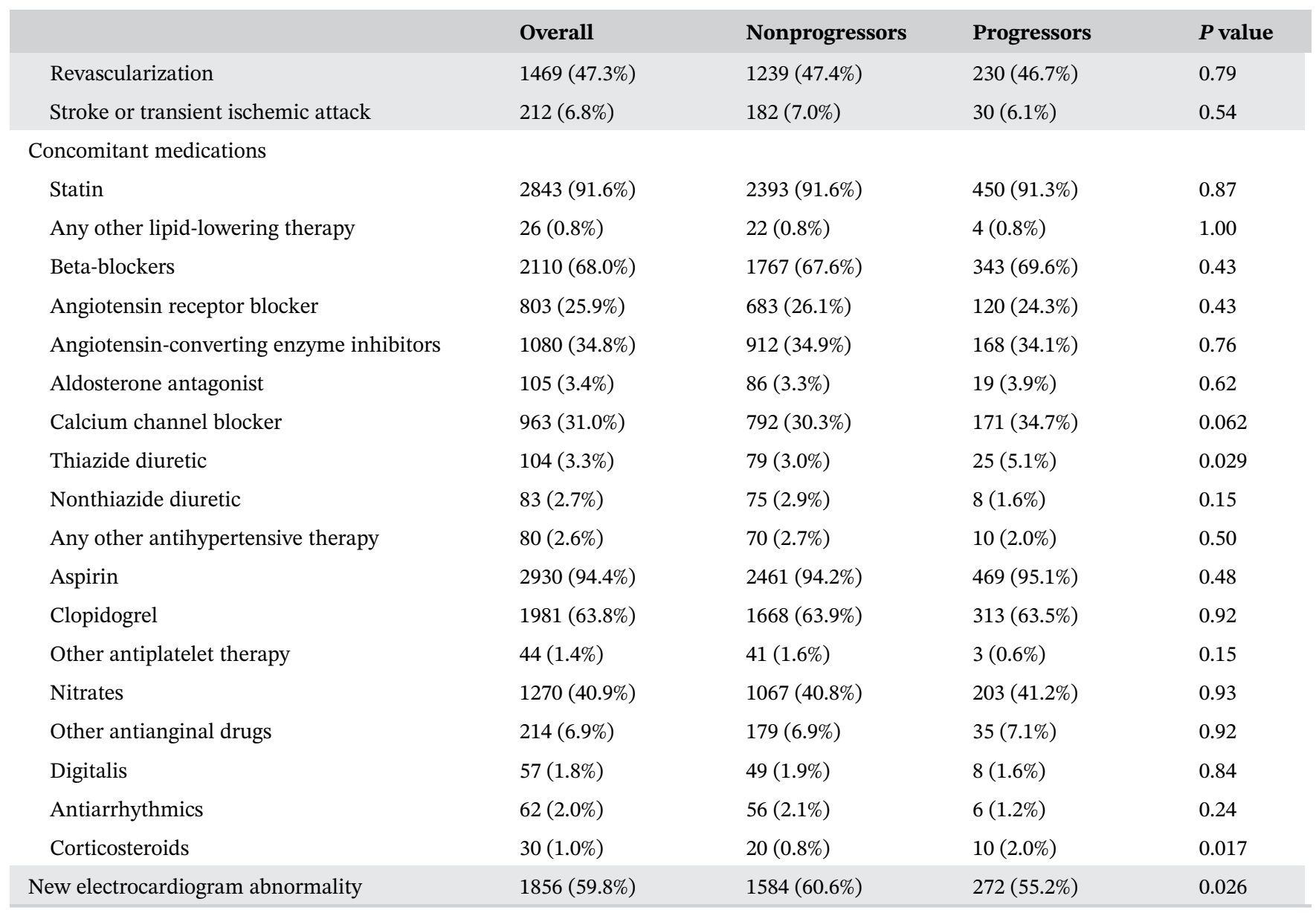

Note: Data are shown as median and interquartile range or $\mathrm{N}(\%)$.

\begin{tabular}{|c|c|c|c|c|c|}
\hline \multicolumn{2}{|l|}{ (A) } & \multicolumn{2}{|l|}{ (B) } & \multicolumn{2}{|l|}{ (C) } \\
\hline \multicolumn{2}{|r|}{ BASIC Model } & \multicolumn{2}{|r|}{ EXTENDED Model } & \multicolumn{2}{|r|}{ FULL Model } \\
\hline$\beta_{\mathrm{n}}$ & $x_{n}$ & & $x_{n}$ & $\beta_{\mathrm{n}}$ & $x_{n}$ \\
\hline-2.831 & (Intercept) & -14.499 & (Intercept) & -14.619 & (Intercept) \\
\hline-0.022 & Age (years) & -0.023 & Age (years) & -0.022 & Age (years) \\
\hline 0.098 & BMI (kg/m2) & 0.317 & Sex $($ Male=1, Female $=0)$ & 0.328 & Sex $($ Male=1, Female $=0)$ \\
\hline 1.095 & Taking Corticosteroids $(\mathrm{Yes}=1, \mathrm{No}=0$ ) & 0.068 & BMI (kg/m2) & 0.069 & BMI (kg/m2) \\
\hline 0.617 & Taking Thiazide diuretic (Yes=1, No=0) & 1.081 & Taking Corticosteroids (Yes=1, No=0) & 1.036 & Taking Corticosteroids (Yes=1, No=0) \\
\hline \multirow{2}{*}{ (D) } & & 0.539 & Taking Thiazide Diuretic $\left(Y e s=1, N_{0}=0\right)$ & 0.534 & Taking Thiazide diuretic $(\mathrm{Yes}=1, \mathrm{No}=0)$ \\
\hline & 1 & 0.739 & $\mathrm{FPG}(\mathrm{mmol} / \mathrm{L})$ & 0.744 & FPG (mmol/L) \\
\hline$P=$ & & 0.482 & $2 \mathrm{hPG}(\mathrm{mmol} / \mathrm{L})$ & 0.489 & $2 \mathrm{hPG}(\mathrm{mmol} / \mathrm{L})$ \\
\hline \multirow{2}{*}{\multicolumn{2}{|c|}{$1+e^{-\left(\beta_{0}+\beta_{1} x_{1}+\beta_{2} x_{2}+\cdots+\beta_{n} x_{n}\right)}$}} & 0.596 & $\mathrm{HbA1c}(\%)$ & 0.615 & HbA1c (\%) \\
\hline & & & & -0.356 & ECG abnormality $\left(\right.$ Yes $\left.=1, \mathrm{No}_{0}=0\right)$ \\
\hline
\end{tabular}

F I G U RE 1 Intercepts and ß coefficients for the BASIC (A), EXTENDED (B), and FULL (C) models, and the model equation (D) using these values to estimate the probability of developing diabetes within 5 years. BMI, body mass index; ECG, electrocardiogram; FPG, fasting plasma glucose; 2hPG, 2-hour plasma glucose

diabetes, of whom 1088 had complete data for the variables required to perform external validation of our final 5-year diabetes risk prediction model and 5-year diabetes risk score algorithm. 


\section{3 | RESULTS}

\section{1 | Population}

Of 3250 ACE placebo-assigned participants, 3105 (96\%) had the requisite data for our analyses. Their baseline characteristics are listed in Table 1. Participants were predominantly Han (96.7\%) and male (72.4\%), with median age 63.0 years, BMI $25.4 \mathrm{~kg} / \mathrm{m}^{2}$, FPG $5.4 \mathrm{mmol} / \mathrm{L}$, $2 \mathrm{hPG}$ $9.1 \mathrm{mmol} / \mathrm{L}$, and $\mathrm{HbAlc} 5.9 \%$ (41 mmol/mol). CHD history was categorized (not mutually exclusively) as myocardial infarction (44.0\%), unstable angina (42.9\%), or stable angina (22.1\%), with most participants having a prior history of hypertension (65.5\%) and taking statins (91.6\%), beta-blockers (68.0\%), and aspirin (94.4\%).

Over median 5.0 years follow-up, 493 (15.9\%) ACE participants progressed to diabetes. Compared with nonprogressors, progressors were more likely to be younger, male, and current smokers, with higher adiposity, glucose, and lipid measures, and to have a prior history of hypertension or atrial fibrillation and to be taking thiazide diuretics or corticosteroids (Table 1).

\section{2 | Five-Year diabetes risk prediction model}

The univariate associations with new-onset diabetes for 55 ACE candidate variables are summarized in Table S1. Of these, the 33 that were clinically relevant or had nominally significant associations with new-onset diabetes, were used for model development. Interactions between sex and other covariates did not achieve statistical significance (sex and age, $P=0.055$; sex and BMI, $P=0.056)$ and were not included in the models.

In the BASIC model (Figure 1A), major risk factors for new-onset diabetes were lower age, higher BMI, and use of corticosteroids or thiazide diuretics. Modeling these variables yielded a C-statistic of 0.610 (Table 2) with a good fit $(P=0.84)$ (Figure S1A).

In the EXTENDED model (Figure 1B), the risk factors for new-onset diabetes added to the BASIC model were male sex and a higher baseline FPG, 2hPG, and HbA1c. Their inclusion increased the C-statistic from 0.610 to 0.757 (Table 2) and yielded a good fit $(P=0.20)$ (Figure S1B).

In the FULL model (Figure 1C), a new electrocardiographic abnormality was associated with a lower risk of diabetes incidence, but adding this variable only minimally improved the C-statistic from 0.757 to 0.761 (Table 2), also with a good fit $(P=0.47)$ (Figure S1C).

Accordingly, we elected to use the EXTENDED model as its performance was similar to the FULL model and because the variables required are more readily available.

\section{3 | Five-year T2D risk score algorithm}

We constructed a risk scoring algorithm using the EXTENDED model equations that produced risk scores ranging from 0 to 23 points (Figure 2). When the risk score was applied to ACE placebo participants it yielded a C-statistic of 0.754 with a good of fit $(P=0.58)$ (Figure S1D). The proportions of the ACE placebo population classified by the risk scoring algorithm as high, moderate and modest risk were $2.9 \%, 16.2 \%$, and $80.8 \%$

T A B L E 2 Five-year multivariable diabetes risk prediction models for Chinese people with CHD and IGT (N = 3105, 493 events)

\begin{tabular}{|c|c|c|c|c|c|c|}
\hline Model variables & \multicolumn{2}{|l|}{ BASIC model } & \multicolumn{2}{|c|}{ EXTENDED model } & \multicolumn{2}{|l|}{ FULL model } \\
\hline Age (per 10-year increase) & $0.80(0.70-0.91)$ & .00060 & $0.79(0.69-0.91)$ & .00096 & $0.80(0.70-0.92)$ & 0.0017 \\
\hline Male (vs female) & - & - & $1.37(1.08-1.76)$ & .011 & $1.39(1.09-1.78)$ & 0.0085 \\
\hline Body mass index (per 1 kg/m²) & $1.10(1.07-1.14)$ & $<.0001$ & $1.07(1.03-1.11)$ & $<.0001$ & $1.07(1.04-1.11)$ & $<0.0001$ \\
\hline Fasting plasma glucose (per $1 \mathrm{mmol} / \mathrm{L}$ ) & - & - & $2.09(1.77-2.48)$ & $<.0001$ & $2.10(1.78-2.49)$ & $<0.0001$ \\
\hline 2-hour plasma glucose (per $1 \mathrm{mmol} / \mathrm{L})$ & - & - & $1.62(1.46-1.80)$ & $<.0001$ & $1.63(1.47-1.81)$ & $<0.0001$ \\
\hline HbA1c (per 1\%) & - & - & $1.81(1.56-2.11)$ & $<.0001$ & $1.85(1.59-2.15)$ & $<0.0001$ \\
\hline Electrocardiogram abnormality (vs normal) & - & - & - & - & $0.70(0.57-0.87)$ & 0.00093 \\
\hline
\end{tabular}

Abbreviations: 95\% CI, 95\% confidence interval; CHD, coronary heart disease; IGT, impaired glucose tolerance; OR, odds ratio. 


\begin{tabular}{|c|c|}
\hline \multicolumn{2}{|c|}{ Step 1. Sum scores for each risk factor } \\
\hline Risk Factor & Points \\
\hline \multicolumn{2}{|l|}{ Age (years) } \\
\hline$\leq 60$ & 2 \\
\hline $60-80$ & 1 \\
\hline$\geq \mathbf{8 0}$ & 0 \\
\hline \multicolumn{2}{|l|}{ Sex } \\
\hline Female & 0 \\
\hline Male & 1 \\
\hline \multicolumn{2}{|c|}{ Body mass index $\left(\mathrm{kg} / \mathrm{m}^{2}\right)$} \\
\hline$<24$ & 0 \\
\hline$\geq 24$ to $<28$ & 1 \\
\hline$\geq 28$ & 2 \\
\hline \multicolumn{2}{|c|}{ Taking corticosteroids } \\
\hline No & 0 \\
\hline Yes & 4 \\
\hline \multicolumn{2}{|c|}{ Taking thiazide diuretics } \\
\hline No & 0 \\
\hline Yes & 2 \\
\hline \multicolumn{2}{|c|}{ Fasting plasma glucose $(\mathrm{mmol} / \mathrm{L})$} \\
\hline$<5.6$ & 0 \\
\hline$\geq 5.6$ to $<6.1$ & 2 \\
\hline$\geq 6.1$ to 7.0 & 4 \\
\hline \multicolumn{2}{|c|}{ 2-hour plasma glucose (mmol/L) } \\
\hline 7.8 to $<8.6$ & 0 \\
\hline$\geq 8.6$ to $<9.4$ & 1 \\
\hline$\geq 9.4$ to $<10.2$ & 3 \\
\hline$\geq 10.2$ to 11.1 & 4 \\
\hline \multicolumn{2}{|c|}{$\mathrm{HbA}_{\mathrm{lc}}(\%)(\mathrm{mmol} / \mathrm{mol})$} \\
\hline$<5.7(<39)$ & 0 \\
\hline $\begin{array}{l}\geq 5.7 \text { to }<6.5 \\
\geq 39 \text { to }<48)\end{array}$ & 2 \\
\hline$\geq 6.5(\geq 48)$ & 4 \\
\hline
\end{tabular}

\begin{tabular}{|c|c|}
\hline \multicolumn{2}{|c|}{$\begin{array}{l}\text { Step 2. Read off five-year } \\
\text { diabetes risk corresponding } \\
\text { to the total score }\end{array}$} \\
\hline Total Score & $\begin{array}{c}\text { Five-year risk } \\
\text { of diabetes }\end{array}$ \\
\hline 0 & $1 \%$ \\
\hline 1 & $2 \%$ \\
\hline 2 & $3 \%$ \\
\hline 3 & $4 \%$ \\
\hline 4 & $5 \%$ \\
\hline 5 & $6 \%$ \\
\hline 6 & $\mathbf{8} \%$ \\
\hline 7 & $11 \%$ \\
\hline 8 & $14 \%$ \\
\hline 9 & $18 \%$ \\
\hline 10 & $23 \%$ \\
\hline 11 & $29 \%$ \\
\hline 12 & $35 \%$ \\
\hline 13 & $42 \%$ \\
\hline 14 & $50 \%$ \\
\hline 15 & $57 \%$ \\
\hline 16 & $65 \%$ \\
\hline 17 & $71 \%$ \\
\hline 18 & $77 \%$ \\
\hline 19 & $82 \%$ \\
\hline 20 & $86 \%$ \\
\hline 21 & $89 \%$ \\
\hline 22 & $92 \%$ \\
\hline 23 & $94 \%$ \\
\hline
\end{tabular}

F I G U RE 2 Risk scoring algorithm based on EXTENDED model equations for estimating 5-year diabetes risk

respectively, with $52.7 \%, 35.5 \%$, and $10.6 \%$ respectively developing diabetes (Figure 3).

\subsection{External validation}

Baseline characteristics for the 1088 Luzhou survey participants with IGT are listed in Table S2. They were all of Han ethnicity, more often female (66.8\%), with a median age of 60.0 years, and the majority (96.3\%) had no history of CHD. Overall, $230(21.1 \%$, 95\% conference interval [CI] $18.8 \%$ - $23.7 \%$ ) participants progressed to T2D over a median of 3.0 years, with a higher incidence in those with a history of CHD (13 of 40, 32.5\%). To obtain an estimated 5-year diabetes incidence for the Luzhou survey cohort we applied a linear extrapolation, multiplying the observed incidence by $5 / 3$ to give 383 progressors (35.2\%, 95\% CI $31.3 \%$ - 39.5\%).

The EXTENDED model predicted a 5-year diabetes incidence of $13.9 \%$ for the Luzhou survey cohort, 


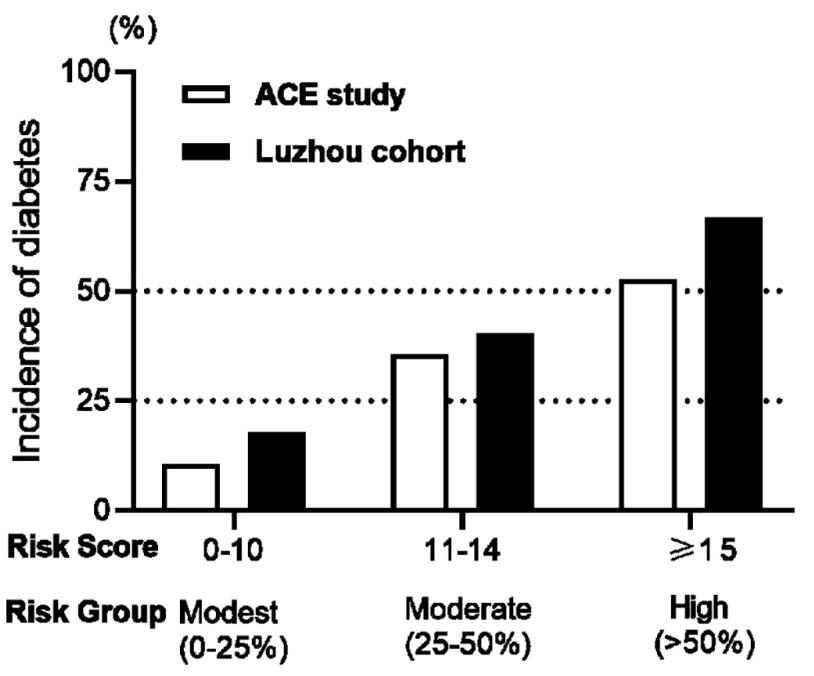

F I G URE 3 Diabetes progression in three risk classes (using risk score algorithm) in Acarbose Cardiovascular Evaluation (ACE) study and Luzhou survey cohort

substantially less than the projected proportion, with a C-statistic of 0.643 (0.602-0.685). The risk scoring algorithm predicted a 5 -year diabetes incidence of $13.7 \%$, with a C-statistic of 0.638 (0.595-0.680). The risk scoring algorithm classified $0.8 \%, 13.0 \%$, and $86.2 \%$ of Luzhou survey cohort participants as high, moderate, and modest risk respectively, with $66.7 \%, 40.4 \%$, and $17.8 \%$ respectively progressing to diabetes (Figure 3).

\section{4 | DISCUSSION}

We have developed a risk calculator, in compliance with the TRIPOD statement, ${ }^{22}$ that predicts the 5 -year likelihood that Chinese people with CHD and IGT will develop diabetes. This calculator uses variables that are readily available in an outpatient setting and can be used either in the form of equations or as a risk scoring algorithm.

The BASIC model, which just required information on age, BMI, and whether or not a patient was taking corticosteroids or a thiazide diuretics, had only moderate discrimination. The EXTENDED model, which added sex, baseline FPG, 2hPG, and HbA1c, showed good discrimination and a good fit. In the FULL model the only additional variable to be included was a new electrocardiographic abnormality but this did not materially alter the C-statistic or goodness of fit. Accordingly, we recommend using the EXTENDED model equations or the risk scoring algorithm derived from it which performed equally well.

Baseline variables shown here to be significantly associated with diabetes incidence are largely consistent with those in other diabetes prediction models. Higher BMI, male sex, use of corticosteroids, or thiazide diuretics and higher glucose levels are strongly associated with increased diabetes risk. ${ }^{25-31}$ Among all variables, the three glucose measures (FPG, 2hPG, and HbA1c) were the most powerful predictors for future diabetes incidence. Our study found that lower age was associated with higher risk of diabetes, contrary to the trend in normal glucose tolerance populations but consistent with previous IGT population findings. ${ }^{12}$ Developing IGT at earlier age may indicate that this population carries a stronger genetic background related to diabetes, or more unfavorable environmental and lifestyle factors, and may therefore be associated with a more rapid progression to diabetes. We have no explanation for the finding that a new electrocardiogram abnormality (including clinically insignificant and significant abnormality) at baseline was significantly associated with a reduced risk of diabetes.

External validation of the EXTENDED model and the risk scoring algorithm in the Luzhou survey cohort of Chinese individuals with IGT $^{24}$ showed acceptable discrimination but substantially underestimated diabetes incidence in this population. This may be because only $3.7 \%$ of the Luzhou survey cohort had CHD as well as IGT. Also, compared with the ACE study population, the Luzhou survey cohort population were younger and had a lower proportion of males, current smokers, current alcohol users, prior hypertension, with very few taking statins, a lower BMI, and a higher low-density lipoprotein cholesterol. These differences between the two populations are largely consistent with the differences between people with and without CHD. Further external validation, and possibly calibration, will be required to maximize the model's predictive ability.

Previous European and Chinese surveys have shown a high prevalence of IGT among CHD patients, approximately $32 \%$ to $33 \%$ respectively. ${ }^{16,17}$ Those patients who progressed to diabetes have been reported to be at higher risk of adverse clinical outcomes with a greater mortality rate than those who do not develop diabetes. ${ }^{32,33}$ Using our T2D risk calculator could provide individualized risk estimates for Chinese patients with CHD and IGT, which in turn could enhance their awareness of T2D risk and prompt interventions that may prevent or delay the onset of T2D in this population.

Categorizing estimated 5-year diabetes risk as "modest risk" (0\%-25\%), "moderate" (>25\%-50\%), or "high" $(>50 \%)$ provides a simple stratification that could help select an appropriate intensity of intervention. Possible recommendations might be routine lifestyle advice for those at modest risk, intensive lifestyle intervention for those at moderate risk and intensive lifestyle plus pharmacologic intervention for those at high risk of 
developing diabetes. Thus in clinical practice personalized diabetes risk estimates and risk classification could assist clinicians and patients when discussing the need to initiate primary prevention measures and the intensity required. External validation in Luzhou survey cohort showed that our model may have acceptable risk stratification ability in other IGT populations, which may help inform decision-making when considering primary prevention in this population.

Our study has several strengths. The ACE trial was a well-designed, large-scale, long-term secondary cardiovascular prevention study for which OGTT-confirmed new-onset diabetes was a prespecified secondary outcome, making it an excellent resource to evaluate baseline predictors of the development of new-onset diabetes. Also, as it was a multicenter study recruiting subjects from 176 sites across mainland China and Hong Kong, participants were likely to be representative of the Chinese population as whole with CHD and IGT.

The study also has several limitations. First, the ACE trial did not collect previously well-recognized predictors of diabetes such as a family history of diabetes and lifestyle and dietary factors (physical activity, fruit and vegetable consumption). Second, as the overwhelming majority of ACE population were taking the guideline recommend drugs for CHD management, it was not possible to test whether any of them individually had an impact on T2D risk, for example, aspirin or statins. Third, we could validate our model at this time only in a Chinese IGT population most of whom did not have CHD. Fourth, ACE trial participants were recruited from toplevel Chinese hospitals (tier 2 and tier 3) meaning there was likely a selection bias to the population studied. Fifth, all ACE trial subjects received appropriate lifestyle advice with respect to diet, exercise, and smoking and their cardiovascular therapy was optimized, which may have led to a lower diabetes incidence than might otherwise have been expected.

In conclusion, in Chinese people with CHD and IGT, lower age, male sex, obesity, use of corticosteroids, or thiazide diuretics as well as higher FPG, 2hPG, and HbA1c were major determinants of new-onset diabetes. A risk prediction model, using routinely available clinical variables and glycemic measures, can estimate T2D risk in Chinese people with CHD and IGT. With further calibration our simple risk calculator could inform decisionmaking when considering primary prevention T2D measures in this population.

\section{ACKNOWLEDGEMENTS}

The ACE trial was conducted by the University of Oxford Diabetes Trial Unit as an academically independent study with funding from Bayer AG. R.R.H. is an Emeritus
National Institute for Health Research Senior Investigator. We thank Qin Wan for access to data from the Luzhou survey cohort.

\section{DISCLOSURE}

S.X., C.S., and R.L.C have no disclosures. J.T. reports research grants and fees for consultancy, advisory board membership, or lectures from AstraZeneca, Bayer AG (related to the present study), Boehringer Ingelheim, Eli Lilly, Impeto Medical, Merck (Germany), Merck Sharp \& Dohme, Sanofi Aventis, Novartis, Novo Nordisk, and Servier. R.R.H. reports research support from AstraZeneca, Bayer, and Merck Sharp \& Dohme and personal fees from Bayer, Intarcia, Merck Sharp \& Dohme, Novartis, and Novo Nordisk.

\section{ORCID}

\section{Shishi Xu (1) https://orcid.org/0000-0003-1696-2178}

\section{REFERENCES}

1. International Diabetes Federation. IDF Diabetes Atlas. 9th ed. Brussels, Belgium: International Diabetes Federation; 2019 .

2. Wang L, Gao P, Zhang M, et al. Prevalence and ethnic pattern of diabetes and Prediabetes in China in 2013. JAMA. 2017;317: 2515-2523.

3. Xu Y, Wang L, He J, et al. Prevalence and control of diabetes in Chinese adults. JAMA. 2013;310:948-959.

4. Pan XR, Li GW, Hu YH, et al. Effects of diet and exercise in preventing NIDDM in people with impaired glucose tolerance. The Da Qing IGT and diabetes study. Diabetes Care. 1997;20: 537-544.

5. Knowler WC, Barrett-Connor E, Fowler SE, et al. Reduction in the incidence of type 2 diabetes with lifestyle intervention or metformin. N Engl J Med. 2002;346:393-403

6. Tuomilehto J, Lindstrom J, Eriksson JG, et al. Prevention of type 2 diabetes mellitus by changes in lifestyle among subjects with impaired glucose tolerance. N Engl J Med. 2001;344:13431350.

7. Chiasson JL, Josse RG, Gomis R, Hanefeld M, Karasik A, Laakso M. Acarbose for prevention of type 2 diabetes mellitus: the STOP-NIDDM randomised trial. Lancet. 2002;359:20722077.

8. Zinman B, Harris SB, Neuman J, et al. Low-dose combination therapy with rosiglitazone and metformin to prevent type 2 diabetes mellitus (CANOE trial): a double-blind randomised controlled study. Lancet. 2010;376:103-111.

9. Armato JP, DeFronzo RA, Abdul-Ghani M, et al. Successful treatment of prediabetes in clinical practice using physiological assessment (STOP DIABETES). Lancet Diabetes Endocrinol. 2018;6:781-789.

10. Holman RR, Coleman RL, Chan JCN, et al. Effects of acarbose on cardiovascular and diabetes outcomes in patients with coronary heart disease and impaired glucose tolerance (ACE): a randomised, double-blind, placebo-controlled trial. Lancet Diabetes Endocrinol. 2017;5:877-886.

11. Li G, Zhang P, Wang J, et al. Cardiovascular mortality, allcause mortality, and diabetes incidence after lifestyle 
intervention for people with impaired glucose tolerance in the Da Qing diabetes prevention study: a 23-year follow-up study. Lancet Diabetes Endocrinol. 2014;2:474-480.

12. Bethel MA, Chacra AR, Deedwania P, et al. A novel risk classification paradigm for patients with impaired glucose tolerance and high cardiovascular risk. Am J Cardiol. 2013;112:231-237.

13. Tuomilehto J, Lindstrom J, Hellmich M, et al. Development and validation of a risk-score model for subjects with impaired glucose tolerance for the assessment of the risk of type 2 diabetes mellitus-the STOP-NIDDM risk-score. Diabetes Res Clin Pract. 2010;87:267-274.

14. Yoon KH, Lee JH, Kim JW, et al. Epidemic obesity and type 2 diabetes in Asia. Lancet. 2006;368:1681-1688.

15. Ramachandran A, Ma RC, Snehalatha C. Diabetes in Asia. Lancet. 2010;375:408-418.

16. Bartnik M, Ryden L, Ferrari R, et al. The prevalence of abnormal glucose regulation in patients with coronary artery disease across Europe. The Euro Heart Survey on diabetes and the heart. Eur Heart J. 2004;25:1880-1890.

17. Hu DY, Pan CY, Yu JM, the China Heart Survey Group. The relationship between coronary artery disease and abnormal glucose regulation in China: the China Heart Survey. Eur Heart J. 2006;27:2573-2579.

18. Holman RR, Bethel MA, Chan JC, et al. Rationale for and design of the acarbose cardiovascular evaluation (ACE) trial. Am Heart J. 2014;168:23-29. e22.

19. Theodorakis MJ, Coleman RL, Feng H, et al. Baseline characteristics and temporal differences in acarbose cardiovascular evaluation (ACE) trial participants. Am Heart J. 2018;199:170-175.

20. SAS Institute (2013) SAS Version 9.3. SAS Inst., Cary.

21. R Core Team. R: A Language and Environment for Statistical Computing. Vienna, Austria: R Foundation for Statistical Computing; 2017. https://www.R-project.org/.

22. Collins GS, Reitsma JB, Altman DG, et al. Transparent reporting of a multivariable prediction model for individual prognosis or diagnosis (TRIPOD): the TRIPOD statement. BMJ. 2015;350:g7594.

23. Chen C, Lu FC. Department of Disease Control Ministry of Health PRC. The guidelines for prevention and control of overweight and obesity in Chinese adults. Biomed Environ Sci. 2004;17:1-36.

24. Bi Y, Lu J, Wang W, et al. Cohort profile: risk evaluation of cancers in Chinese diabetic individuals: a longitudinal (REACTION) study. J Diabetes. 2014;6:147-157.

25. Schmidt MI, Duncan BB, Bang H, et al. Identifying individuals at high risk for diabetes: the atherosclerosis risk in communities study. Diabetes Care. 2005;28:2013-2018.
26. Chen L, Magliano DJ, Balkau B, et al. AUSDRISK: an Australian type 2 diabetes risk assessment tool based on demographic, lifestyle and simple anthropometric measures. Med J Aust. 2010;192:197-202.

27. Rahman M, Simmons RK, Harding AH, Wareham NJ, Griffin SJ. A simple risk score identifies individuals at high risk of developing type 2 diabetes: a prospective cohort study. Fam Pract. 2008;25:191-196.

28. Lindstrom J, Tuomilehto J. The diabetes risk score: a practical tool to predict type 2 diabetes risk. Diabetes Care. 2003;26:725-731.

29. Wilson PW, Meigs JB, Sullivan L, Fox CS, Nathan DM, Sr D'Agostino RB. Prediction of incident diabetes mellitus in middle-aged adults: the Framingham offspring study. Arch Intern Med. 2007;167:1068-1074.

30. Stern MP, Williams K, Haffner SM. Identification of persons at high risk for type 2 diabetes mellitus: do we need the oral glucose tolerance test? Ann Intern Med. 2002;136: 575-581.

31. Hippisley-Cox J, Coupland C. Development and validation of QDiabetes-2018 risk prediction algorithm to estimate future risk of type 2 diabetes: cohort study. BMJ. 2017;359:j5019.

32. Eitel I, Hintze S, de Waha S, et al. Prognostic impact of hyperglycemia in nondiabetic and diabetic patients with ST-elevation myocardial infarction: insights from contrast-enhanced magnetic resonance imaging. Circ Cardiovasc Imaging. 2012;5:708-718.

33. Reinstadler SJ, Stiermaier $\mathrm{T}$, Eitel $\mathrm{C}$, et al. Relationship between diabetes and ischaemic injury among patients with revascularized ST-elevation myocardial infarction. Diabetes Obes Metab. 2017;19:1706-1713.

\section{SUPPORTING INFORMATION}

Additional supporting information may be found online in the Supporting Information section at the end of this article.

How to cite this article: $\mathrm{Xu} S$, Scott $\mathrm{CAB}$, Coleman RL, Tuomilehto J, Holman RR. Predicting the risk of developing type 2 diabetes in Chinese people who have coronary heart disease and impaired glucose tolerance. Journal of Diabetes. 2021;13:817-826. https://doi.org/10. 1111/1753-0407.13175 